\title{
Sodium Alginate and Gelatin Hydrogels: Viscosity Effect on Hydrophobic Drug Release
}

\author{
Utkarsh Bhutani, Anindita Laha, Kishalay Mitra, and Saptarshi Majumdar*
}

Department of Chemical Engineering, Indian Institute of Technology, Hyderabad, INDIA

${ }^{*} E$-mail: saptarshi@iith.ac.in

\begin{abstract}
Blend of biodegradable hydrogels like sodium alginate/gelatin (SA/G) usually requires use of chemical cross-linkers to remain stable in aqueous media for drug delivery applications. This study targets the feasibility of having an entire spectrum of a model hydrophobic drug (piperine) release i.e. from burst to controlled release, by varying polymer viscosity and molecular weight of plasticizer with minimal use of cross-linkers. Swelling study, drug-polymer interactions and morphology analysis reveal the impact of viscosity variation on polymer matrix.
\end{abstract}

Keywords: Sodium Alginate, Gelatin, Piperine, Hydrogels, Viscosity, Plasticizer

\section{Introduction}

Biopolymers like starch [1], gelatin [2], alginate [3], chitosan [4] and many others have been exploited in the field of drug delivery. Systems like thin-films [5], micelles [6] etc. prepared using these polymers have been used in the controlled delivery of drugs. Hydrogel is also one of these systems that has been the choice of many researchers in recent times. Their porosity and property to swell make them excellent systems to be utilized in the field of drug delivery [7]. SA and gelatin are biodegradable polymers used in this work. Piperine was used as hydrophobic drug.

In this work, the effect of viscosity on swelling and dissolution of SA/G hydrogels was investigated. The idea here was to impart dissolution stability and achieve a controlled swelling with minimal use of toxic cross-linkers like glutaraldehyde (GTA). It was also observed that viscosity plays a very crucial role in loading and release of piperine. We have also tried to investigate the role of plasticizer like poly ethylene glycol (PEG) to improve the drug 
encapsulation. The overall aim was to achieve a wide spectrum of drug release, i.e. from controlled to burst release by varying polymer viscosity.

\section{Material and Methods}

All chemicals were obtained from Alfa Aesar. SA/G hydrogels were prepared with few modification to the available method [8] i.e. use of HV (high viscosity) SA (1000-1500 cps, $1 \%$ in water) and the preparation of hybrid hydrogels (HV and LV-low viscosity SA in 1:1 ratio i.e. a 60/40 hybrid hydrogel has $30 \%$ of each HV and LV SA along-with $40 \%$ gelatin w/w). Apart from PEG 2000; 4000 and 6000 were also used. GTA was used as a cross-linker (0.2 v/v for $10 \mathrm{~min}$ ) and $25 \mathrm{mg}$ of piperine was also added to selected samples as a drug during the preparation of hydrogels. Swelling degree (SD) studies were carried out in PBS (Phosphate buffer saline pH 7.4) and 0.1 N HCl (pH 1.2). The

SD was calculated by the equation: $\mathrm{SD}(\%)=\frac{\boldsymbol{W} \boldsymbol{s}-\boldsymbol{W d}}{\boldsymbol{W d}} \times 100$, where $W_{s}$ is weight of swollen sample, while $W_{d}$ is weight of dry samples. The FTIR-ATR analysis was done over a range of $500 \mathrm{~cm}^{-1}-4000 \mathrm{~cm}^{-1}$ using Bruker Tensor 37 . Morphology of hydrogels was analyzed using a table top SEM (Phenom world ProX). Drug release was studied both in $\mathrm{PBS}$ and $0.1 \mathrm{~N} \mathrm{HCl}$ solution at a temperature of $37^{\circ} \mathrm{C}$ to mimic the intestinal and gastric $\mathrm{pH}$ i.e. 7.4 and 1.2. Samples were analyzed at regular time intervals using UV-VIS spectroscopy (Lambda 35 Perkin Elmer) at $342 \mathrm{~nm}$ i.e. the $\lambda_{\max }$ for piperine.

\section{Results and Discussion}

Swelling Degree: 60/40 and 70/30 (SA/G) cases were chosen as they are stable up-to $360 \mathrm{~min} .80 / 20$ combination could not be chosen as high concentration (thus high viscosity) of HV SA did not allow proper stirring/ mixing of the solution. Dissolution of LV hydrogels began within 30 min making them difficult candidates for swelling analysis.

Results of SD clearly indicate the role of viscosity in improving the dissolution resistance of hydrogels. Dissolution began after $360 \mathrm{~min}$ and $240 \mathrm{~min}$ for HV hydrogels (Fig. 1a) and hybrid hydrogels (Fig. 1b), respectively. The hybrid hydrogels do not show a clear equilibrium due to rapid swelling of LV SA present in it followed by its dissolution. SD was higher in pH 7.4 and increased with the increase in SA (Fig. 1c). High swelling in pH 7.4 is a result of repulsions induced by the ionization of carboxylate group of SA. In $\mathrm{pH}$ 1.2, these groups remain almost unionized. 


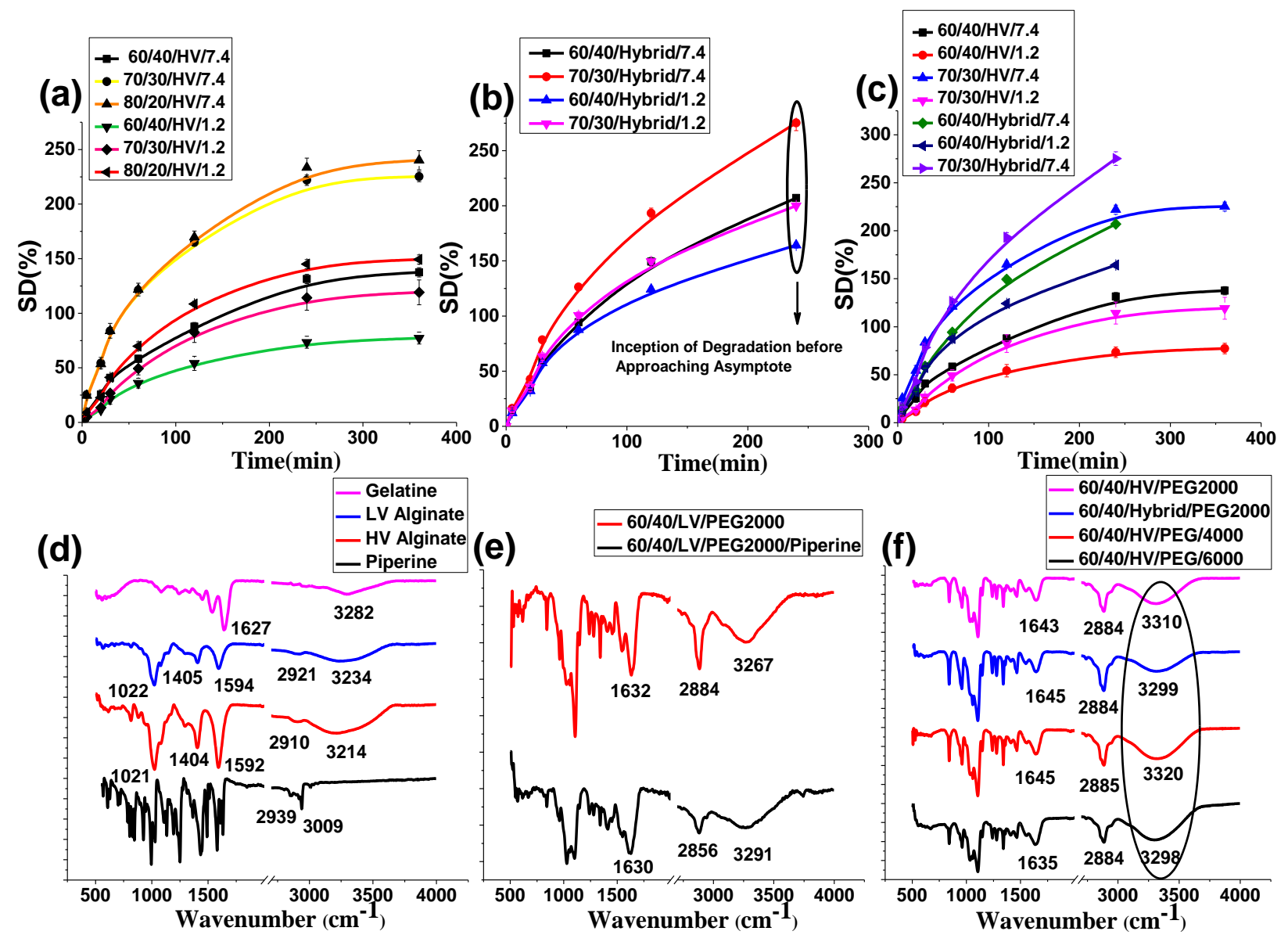

Fig. 1. Swelling Degree (a) 60/40, 70/30 and 80/20 HV hydrogels in pH 7.4/1.2 (b) 60/40 and 70/30 Hybrid hydrogel in pH 7.4/1.2 (c) SD comparison between HV and Hybrid hydrogels. FT-IR/ATR analysis (d) Gelatin, LV SA, HV SA and Piperine (e) 60/40 LV/PEG 2000 and 60/40 LV/PEG2000/Piperine (f) 60/40HV/PEG2000, 60/40 Hybrid/PEG2000, 60/40 HV/PEG4000 and 60/40 HV PEG 6000

FT-IR Analysis: There were marked differences between the spectra of HV and LV SA. It was observed that corresponding stretch values of SA, be it $\mathrm{OH}$ stretch (3234 (LV) and $\left.3214 \mathrm{~cm}^{-1}(\mathrm{HV})\right)$ or CH stretch $(2921(\mathrm{LV})$ and $\left.2910 \mathrm{~cm}^{-1}(\mathrm{HV})\right)$ were less in HV SA (Fig. 1d) as compared to LV SA (Fig. 1d). This tendency was also visible in the spectra of 60/40 LV, HV and hybrid samples where the values corresponding to the $\mathrm{NH}$ stretch were in the order $\mathrm{LV}<<$ Hybrid $<<\mathrm{HV}\left(3267 \mathrm{~cm}^{-1}<<3299 \mathrm{~cm}^{-1}<<3310 \mathrm{~cm}^{-1}\right)$ (Fig. 1e and f). These peaks show a higher tendency of HV SA to form intramolecular hydrogen bonds. The spectra for SA/G hydrogel confirmed the interactions between $\mathrm{NH}$ group of gelatin and $\mathrm{OH}$ group of SA (Fig. 1f) as peak shifts were observed when compared to the spectra of pure SA [3] and gelatin [9] (Fig 1d). 
Stability of piperine inside the hydrogel was confirmed as only minor peak shifts were observed which might have been due to weak association of piperine with SA and gelatin (Fig. 1e). Spectra for hydrogels containing PEG 2000, 4000 and 6000 were also studied. It was observed that PEG places itself between the chains of SA and gelatin, which causes a reduction in intermolecular forces between them. This was confirmed by the increase in peak shift for NHOH in SA/G hydrogels from PEG 2000 to 4000 (Fig. 1f), however the same increase was not observed for PEG 6000. High Mw of PEG may not allow it to diffuse between the polymer chains hence it was unable to show similar plasticizing effect.
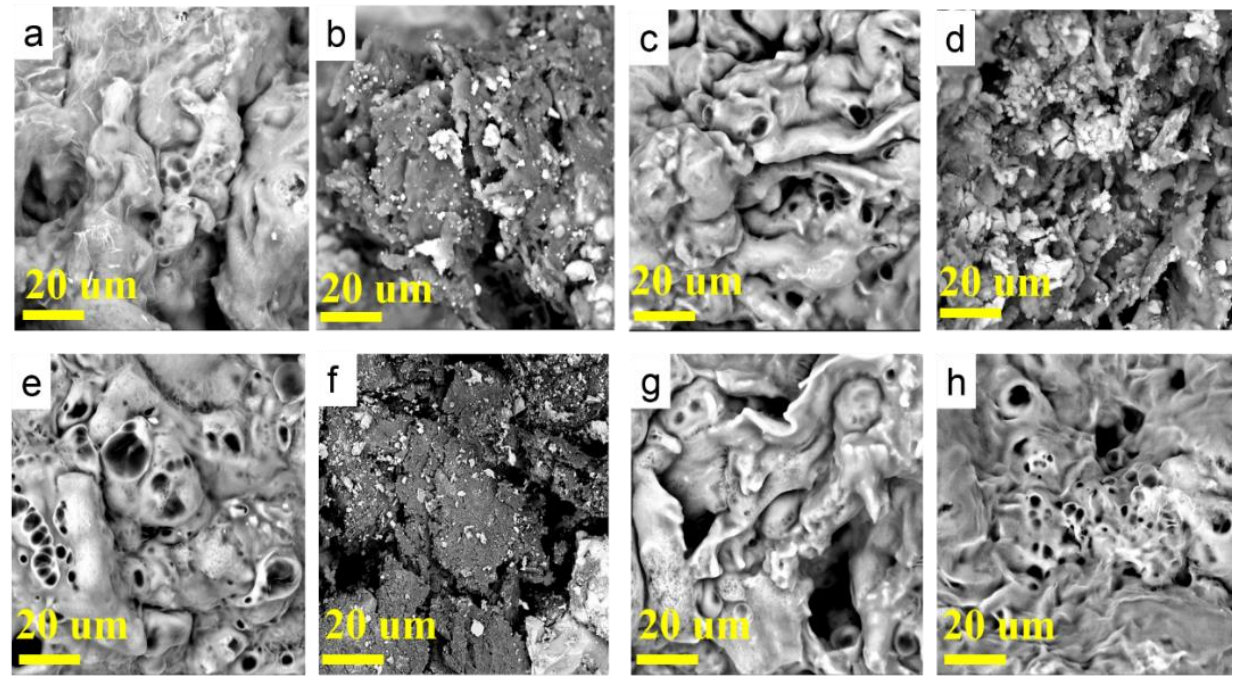

Fig. 2. SEM Analysis: (a) 60/40 LV (b) 60/40 LV 0.2\% GTA (c) 60/40 HV (d) 60/40 HV- 0.2\% GTA (e) 70/30 HV (f) 70/30 HV 0.2\% GTA (g) 70/30 hybrid (h) 60/40 hybrid

Morphology: There were distinct differences observed between 60/40 LV (Fig. 2a), HV (Fig. 2c) hydrogels and their cross-linked counterparts (Fig. $\mathbf{2 b}, \mathbf{d}$ ). Cross-lined samples appear to be rough with the appearance of highly uneven fractured structures like ridges and valleys, while non-cross-linked samples were very smooth in morphology. Similar results were also observed in 70/30 HV cases (Fig. 2e, f). However very distinct changes were not observed in the morphology of LV, HV and hybrid (Fig. 2a, c, g, h) non-cross-linked samples. The LV sample (Fig. 2a) appears to more continuous due to better mixing of SA and gelatin.

Drug Release: The drug release in case of HV hydrogels, was contradicting the results of SD. The drug release in 60/40 HV hydrogel was higher than 70/30 HV although 70/30 had a higher SD. This was due to the early dissolution 
of gelatin and while cross-linked with GTA, its dissolution was controlled. The results now were in accordance with the results of SD (Fig. 3a). LV case (Fig 3b) was accompanied with simultaneous dissolution of both LV SA and gelatin, whereas HV SA could resist dissolution up-to $360 \mathrm{~min}$. The influence of viscosity on drug encapsulation was confirmed by the release in $\mathrm{pH}$ 1.2. Higher burst release was observed in the first $360 \mathrm{~min}$ for the HV hydrogels (12.6 mg) (Table. 1) in pH 1.2 (Fig. 3d) while it was much less in LV hydrogels (4.12 mg) (Fig 3d) (Table. 1). The LV hydrogels due to their low viscosity allowed the drug to diffuse into the core of the hydrogel which was not the case in HV hydrogels. The release in the hybrid hydrogels was anyway higher than LV but lesser than HV in pH 1.2 due to its intermediate viscosity. In order to improve their drug encapsulation, PEG 4000 and 6000 were used to lower the viscosity by increasing the fluidity of the system. It was observed that the burst release was less for HV PEG 4000 case (fig 3e and f) compared to HV PEG 2000 case (Table. 1) for the first 120 min however PEG 6000 could not provide an efficient control over burst release due to its high Mw.

Table 1. Comparison of drug release

\begin{tabular}{|c|c|c|c|c|c|c|}
\hline Time $\longrightarrow$ & \multicolumn{2}{|c|}{$\begin{array}{l}\text { Drug release }(\mathrm{mg}) \\
120 \mathrm{~min}\end{array}$} & \multicolumn{2}{|c|}{$\begin{array}{l}\text { Drug release (mg) } \\
360 \mathrm{~min}\end{array}$} & \multicolumn{2}{|c|}{$\begin{array}{l}\text { Drug release (mg) } \\
600 \mathrm{~min}\end{array}$} \\
\hline Sample/pH $\longrightarrow$ & 7.4 & 1.2 & 7.4 & 1.2 & 7.4 & 1.2 \\
\hline 60/40 LV PEG2000 & $9.65 \pm 0.82$ & $3.10 \pm 0.17$ & $10.48 \pm 0.73$ & $5.12 \pm .20$ & $11.92 \pm 1.88$ & $6.26 \pm .01$ \\
\hline 60/40 HV PEG2000 & $13.57 \pm .068$ & $11.74 \pm 0.23$ & $13.69 \pm 0.12$ & $12.60 \pm 1.63$ & $14.21 \pm 0.47$ & $13.99 \pm 0.32$ \\
\hline 60/40 HYBRID PEG2000 & $13.43 \pm 0.18$ & $3.74 \pm 0.18$ & $14.59 \pm 1.80$ & $5.87 \pm 0.14$ & $14.97 \pm 1.43$ & $6.85 \pm .023$ \\
\hline 60/40 HV PEG4000 & $11.02 \pm 1.98$ & $6.81 \pm 1.25$ & $14.83 \pm .212$ & $9.84 \pm 1.83$ & $15.32 \pm 1.51$ & $10.80 \pm 1.98$ \\
\hline 60/40 HV PEG6000 & $9.13 \pm 0.89$ & $7.65 \pm 0.77$ & $11.67 \pm 0.74$ & $13.01 \pm 0.64$ & $12.10 \pm 0.48$ & $13.72 \pm 0.25$ \\
\hline
\end{tabular}

Release from HV PEG 4000 was higher than LV and hybrid samples in pH 1.2 (Table 1). Hybrid hydrogel seems to solve both the problems i.e. an early dissolution and a better encapsulation due to the presence of both LV SA (improves drug loading) and HV SA (enhances dissolution resistance). Interestingly, a release study of 600 min was possible for LV hydrogels as they remained undisturbed (Low mechanical disturbance) in the release medium unlike the SD experiments, where one needs to remove them for periodical weighing. 


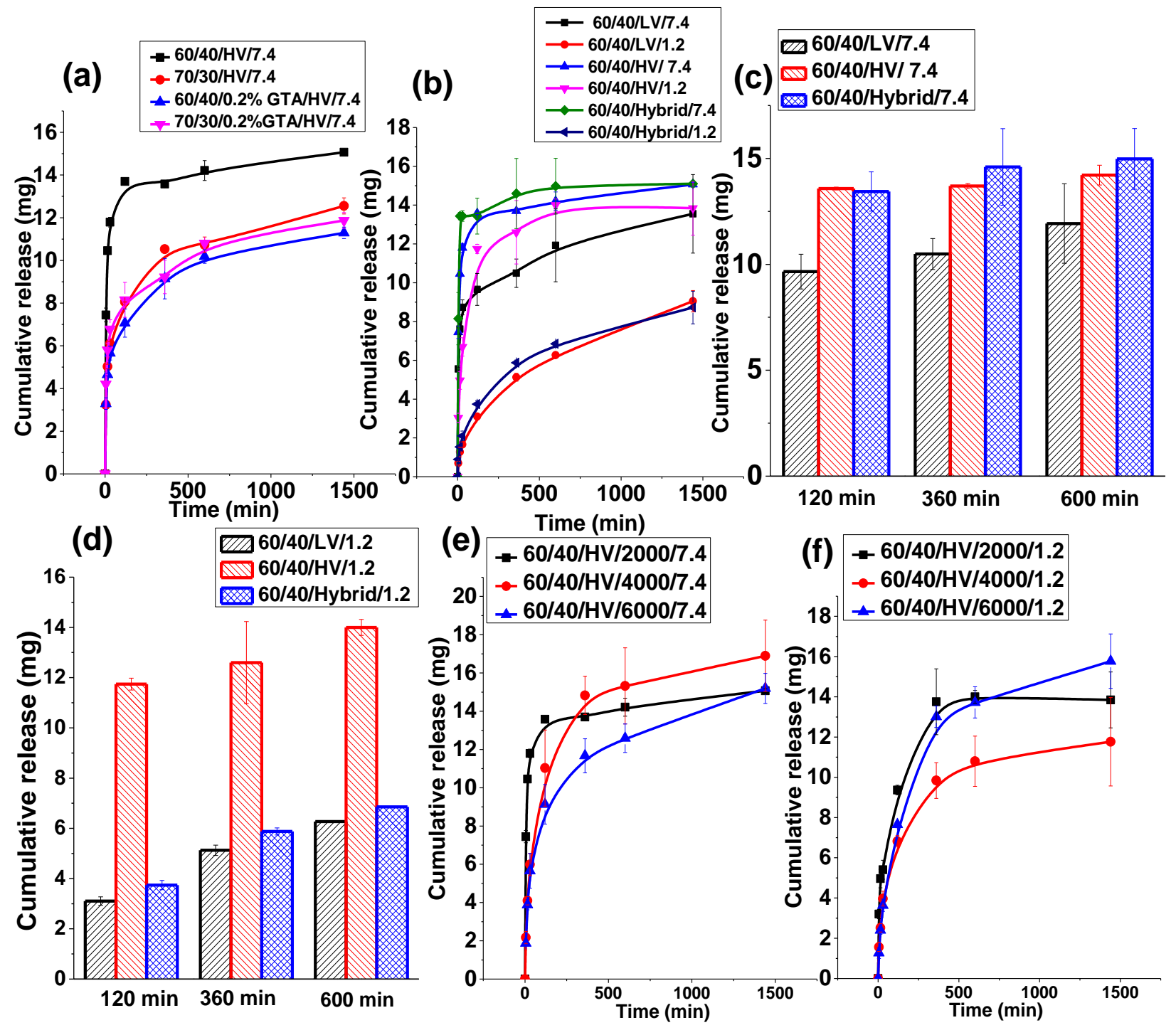

Figure 3. Drug Release (a) 60/40/HV, 70/30 HV, 60/40/0.2\%GTA/HV and 70/30/0.2\%GTA/HV in pH 7.4 (b) 60/40 LV, HV and hybrid samples in $\mathrm{pH} 7.4 / 1.2$ (c) $60 / 40 / \mathrm{LV}$, HV and hybrid release in $\mathrm{pH} 7.4$ at 120,360 and $600^{\text {th }}$ min (d) 60/40/LV, HV and hybrid release in pH 1.2 at 120, 360 and 600 $60^{\text {th }}$ min (e) 60/40/HV PEG 2000/4000/6000 in pH 7.4 (f) 60/40/HV PEG 2000/4000/6000 in pH 1.2

For the real conditions inside our body, LV hydrogels may be used with the cross-linkers like GTA. Finally, hybrid hydrogels offers an escape route where we could achieve dissolution resistance up-to $240 \mathrm{~min}$, a better encapsulation and controlled release without the use of toxic cross-linkers. HV PEG 4000 sample shows promise but reducing the rate of drug release in pH 1.2 still remains a challenge. The rheology study and elaborated explanations to the FT-IR and plasticizing efficiency have been added to the supplementary section. 


\section{Conclusions}

Viscosity had a very important role in increasing the dissolution resistance of SA/G hydrogels from $30 \mathrm{~min}$ in LV to $360 \mathrm{~min}$ and $240 \mathrm{~min}$ in HV and hybrid hydrogels. LV hydrogel were able to encapsulate the drug better followed by hybrid hydrogels. The high burst release of HV hydrogels in $\mathrm{pH} 1.2$ was controlled by increasing the Mw of PEG from 2000 to 4000, while 6000 did not have much effect. Thus the research offers three vehicles that can aid the oral delivery of hydrophobic drugs, (a) hybrid hydrogels that can be used without the use of cross-linker, (b) LV hydrogels that can be cross-linked to avoid early dissolution, (c) HV hydrogels with PEG 4000 that can offer better encapsulation. High release in $\mathrm{pH} 1.2$ for HV hydrogels still remains a challenge.

\section{Acknowledgement}

The authors acknowledge the support of Indian Institute of Technology Hyderabad, for the research facilities.

\section{References}

[1] Elvira C, Mano JF, San Román J, Reis RL. Biomaterials 2002;23:1955-66.

[2] Choi YS, Hong SR, Lee YM, Song KW, Park MH, Nam YS. Biomaterials 1999;20:409-17.

[3] Abd El-Ghaffar M A, Hashem MS, El-Awady MK, Rabie A. M. Carbohydr Polym 2012;89:667-75.

[4] Bernkop-Schnürch Andreas, Sarah Dünnhaupt. Eur. J. Pharm. Biopharm 2012;81:463-69.

[5] Zelikin AN. ACS Nano 2010;4:2494-2509.

[6] Ahmad Z, Shah A, Siddiq M, Kraatz H-B. RSC Adv 2014;4:17028-38.

[7] Hoare TR, Kohane DS. Polymer 2008;49:1993-2007.

[8] Saarai A, Kasparkova V, Sedlacek T, Saha P. J Mech Behav Biomed Mater 2013;18:152-66.

[9] Yin R, Huang Y, Huang C, Tong Y, Tian N. Mater Lett 2009;63:1335-7. 